\title{
Role of nuclear factor kappa $B$ and reactive oxygen species in the tumor necrosis factor- $\alpha$-induced epithelial-mesenchymal transition of MCF-7 cells
}

\section{R. Dong, Q. Wang, \\ X.L. He, Y.K. Chu, \\ J.G. Lu and Q.J. Ma}

Department of General Surgery, Tangdu Hospital,

the Fourth Military Medical University, Xi'an, Shaanxi Province, China

\section{Correspondence \\ J.G. Lu and Q.J. Ma \\ Department of General Surgery \\ Tangdu Hospital \\ Fourth Military Medical University \\ Xi'an 710038, Shaanxi Province \\ China \\ E-mail: maqingjiu@yahoo.com.cn}

Received January 24, 2007 Accepted May 21, 2007

\begin{abstract}
The microenvironment of the tumor plays an important role in facilitating cancer progression and activating dormant cancer cells. Most tumors are infiltrated with inflammatory cells which secrete cytokines such as tumor necrosis factor- $\alpha$ (TNF- $\alpha$ ). To evaluate the role of TNF$\alpha$ in the development of cancer we studied its effects on cell migration with a migration assay. The migrating cell number in TNF- $\alpha$-treated group is about 2-fold of that of the control group. Accordingly, the expression of E-cadherin was decreased and the expression of vimentin was increased upon TNF- $\alpha$ treatment. These results showed that TNF- $\alpha$ can promote epithelial-mesenchymal transition (EMT) of MCF-7 cells. Further, we found that the expression of Snail, an important transcription factor in EMT, was increased in this process, which is inhibited by the nuclear factor kappa B (NFkB) inhibitor aspirin while not affected by the reactive oxygen species (ROS) scavenger $\mathrm{N}$-acetyl cysteine. Consistently, specific inhibition of NFKB by the mutant IKB $\alpha$ also blocked the TNF- $\alpha$-induced upregulation of Snail promoter activity. Thus, the activation of NFKB, which causes an increase in the expression of the transcription factor Snail is essential in the TNF- $\alpha$-induced EMT. ROS caused by TNF- $\alpha$ seemed to play a minor role in the TNF- $\alpha$-induced EMT of MCF-7 cells, though ROS per se can promote EMT. These findings suggest that different mechanisms might be responsible for TNF- $\alpha$ - and ROSinduced EMT, indicating the need for different strategies for the prevention of tumor metastasis induced by different stimuli.
\end{abstract}

Key words

- Tumor necrosis factor- $\alpha$

- Nuclear factor kappa B

- Reactive oxygen species

- Snail

- E-cadherin

- Epithelial-mesenchymal transition

\section{Introduction}

The microenvironment of the tumor plays an important role in facilitating cancer progression and activating dormant cancer cells (1). One feature of many solid tumors is the influx of inflammatory cells, such as tumorinfiltrating lymphocytes and macrophages. These inflammatory cells are assumed to have an important function in the stages of cancer development such as cell transformation, angiogenesis, and metastasis. Indeed, 
focal macrophage infiltration has been linked to increased angiogenesis in human breast and colorectal cancer $(2,3)$. Yet, the functional significance of cytokines produced in situ by inflammatory and tumor cells is still unclear. Another feature of many tumors is the hypoxia of the tumor cells and the resulting production of reactive oxygen species (ROS) $(4,5)$. We reasoned that some of the cytokines released from these cells and hypoxia might serve to enhance the invasive step in breast carcinogenesis through defined signaling pathways. Many epithelial tumors undergo an epithelial-mesenchymal transition (EMT) that facilitates their invasion. It has been reported that either ROS or nuclear factor kappa B (NFkB) could facilitate the EMT in certain cell types (6-10) and tumor necrosis factor- $\alpha$ (TNF- $\alpha$ ), one of the major factors released from the inflammatory cells, could cause NFKB activation and ROS production (11). It has been reported that TNF- $\alpha$ can promote EMT in certain cell types, but the precise mechanism is still unclear (12). Based on the importance of the EMT in carcinoma progression and the prevalence of TNF- $\alpha$ and hypoxia in the presence of tumors, the present study was designed to determine whether TNF- $\alpha$ and ROS are capable of facilitating EMT and to understand the mechanisms involved. We report that MCF-7 cells undergo an EMT conversion from a relatively benign cancer to a migratory phenotype in response to TNF- $\alpha$. Furthermore, our data suggest that this EMT transition is mainly due to the activation of NFKB rather than ROS stimulated by TNF$\alpha$. Overall, our finding has important implications for our understanding of how TNF- $\alpha$ contributes to tumor development.

\section{Material and Methods}

\section{Cell culture and material}

MCF-7 cells, originally obtained from the American Type Culture Collection, were maintained in culture in a $37^{\circ} \mathrm{C}$ incubator with $5 \% \mathrm{CO}_{2}$ in RPMI 1640 medium supplemented with $10 \%$ fetal bovine serum (FBS). All antibodies (anti-vimentin, anti-E-cadherin, anti-actin) used were purchased from Santa Cruz Biotechnology, Inc., Santa Cruz, CA, USA. TNF- $\alpha$ and the ROS-quenching agent $\mathrm{N}$-acetyl cysteine (NAC) were purchased from Sigma (St. Louis, MO, USA).

\section{Cell migration assay}

The cell migration assay was done as previously described (13). MCF-7 cells were grown to $80 \%$ confluence, serum-starved overnight in RPMI 1640 medium and trypsinized and washed in serum-free RPMI 1640 medium before plating onto a 12-well Transwell plate $(12 \mathrm{~mm}$ in diameter, 8 - $\mu \mathrm{m}$ pore size; Corning Incorporated Costar, Lindfield, NSW, Australia). Twelve-well transwell chambers were incubated with RPMI 1640 medium containing $0.01 \%$ bovine serum albumin and $0.01 \%$ FBS overnight. Cells ( $5 \mathrm{x}$ $10^{4}$ ) were added to the upper well, which was placed inside a lower well containing RPMI 1640 medium, 10\% FBS, and various agents indicated in the Results section. After incubation for $24 \mathrm{~h}$, the filter was removed and cells remaining on the upper membrane surface were scraped off. MCF-7 cells that had migrated to the lower side of the filter were fixed in methanol at $4^{\circ} \mathrm{C}$ for $15 \mathrm{~min}$, stained with Toluidine blue and counted under a microscope for quantitation of MCF-7 migration.

\section{RT-PCR}

RT-PCR was performed as previously described (14). At the beginning of each experiment, cells growing in the log phase were plated onto a 6 -well dish at a density of $3 \times 10^{5}$ cells/well and then treated with 10 $\mathrm{ng} / \mathrm{mL}$ TNF- $\alpha, 50 \mu \mathrm{M} \mathrm{H}_{2} \mathrm{O}_{2}, 5 \mathrm{mM}$ aspirin, or $5 \mathrm{mM} \mathrm{NAC}$ or different combinations of these agents. Cells were harvested $24 \mathrm{~h}$ later 
for extraction of RNA, and $2 \mu \mathrm{g}$ total RNA was used to prepare cDNA in a $25-\mu \mathrm{L}$ system (TRizol, Invitrogen, Carlsbad, CA, USA; MLV Reverse transcriptase, Promega, Madison, WI, USA). PCR was then performed on $1 \mu \mathrm{L}$ cDNA with the following primers: Snail forward, GGGCAGGTATGGAGA GGAAGA; Snail reverse, TTCTTCTGCGC TACTGCTGCG; E-cadherin forward, CAG CACGTACACAGCCCTAA; E-cadherin reverse, GCTGGCTCAAGTCAAAGTCC; GAPDH forward, CCTGGCCAAGGTCAT CCATGAC; GAPDH reverse, CATGTAGG CCATGAGGTCCACCAC.

\section{Construction of the Snail promoter reporter and luciferase assay}

A fragment of about $800 \mathrm{bp}$ upstream to the translational start site of Snail was amplified by PCR using the following set of primers: forward CCAGATCTCAAAGCACACT TCCCTTTGCATTG; downward GGGCCA TGGTGGTCGAGGCACTGGGGTC. PCR products were confirmed by sequencing and digested with the indicated restriction nucleases before cloned into the promoterless and enhancerless pGL3 Basic vector (Promega). Cells were plated at a density of $10^{4} /$ well onto a 24-well dish and transiently transfected with $300 \mathrm{ng} 3 \mathrm{X} \kappa \mathrm{B}$ luc (which contains multiple NFKB sites upstream to the minimal promoter-luciferase gene) or Snail reporter or the combination of Snail reporter and the mutant IKB expression vector in the indicated groups. Fifty nanograms of pBIND vector (Promega) was co-transfected as an internal control. Ten nanograms $/ \mathrm{mL}$ TNF$\alpha, 50 \mu \mathrm{M} \mathrm{H}_{2} \mathrm{O}_{2}, 5 \mathrm{mM}$ aspirin, or $5 \mathrm{mM}$ $\mathrm{NAC}$, or different combinations and the control agents were added to the indicated wells $4 \mathrm{~h}$ post-transfection, and cells were harvested $24 \mathrm{~h}$ after the transfection for the luciferase activity test. Experiments for each treatment were performed in triplicate. Luciferase activity was assessed using the Promega luciferase assay system. The luciferase activity of each lysis was measured and then normalized to the activity of Renilla driven by the constitutive expression promoter in the vector of pBIND. Fold induction was calculated relative to the activity observed with the basic pGL3 vector alone.

\section{Western blot analysis}

After stimulation, whole cells were washed with cold phosphate-buffered saline and lysed in $10 \mathrm{mM}$ Tris-HCl, $\mathrm{pH}$ 7.4, 50 $\mathrm{mM} \mathrm{NaCl}, 5 \mathrm{mM}$ ethylenediamine tetraacetic acid, $1 \%$ Nonidet P-40, and $10 \mu \mathrm{g} / \mathrm{mL}$ phenylmethylsulfonyl fluoride. Cell extracts were transferred to microcentrifuge tubes, mixed, and left on ice for $10 \mathrm{~min}$. After one freeze/ thaw cycle, they were centrifuged at 12,000 $g$ for $5 \mathrm{~min}$ at $4^{\circ} \mathrm{C}$. Supernatant samples were subjected to SDS-PAGE and then transferred to nitrocellulose by electrophoresis. Blots were incubated with primary antibodies (antivimentin, anti-E-cadherin, anti-actin) in Trisbuffered saline-Tween-20 plus $2.5 \%$ skim milk overnight. After serial washes with Trisbuffered saline-Tween-20, membranes were incubated with the secondary antibody. Immunoreactive bands were visualized using a peroxidase-conjugated secondary horseradish antibody and subsequent ECL detection (Amersham Pharmacia Biotech, Bucks, UK).

\section{Statistical analysis}

Data are reported as mean \pm SD and the Student $t$-test was applied for statistical analysis, with the level of significance set at $\mathrm{P}<$ 0.05 .

\section{Results}

\section{TNF- $\alpha$ promotes cell migration}

To clarify that TNF- $\alpha$ was involved in the promotion of tumor invasion, we first tested the ability of migration of MCF-7 cells under the treatment used. As shown in 
Figure 1. Tumor necrosis factor- $\alpha$ (TNF- $\alpha)$-induced epithelial-mesenchymal transition in MCF-7 cells. $A$, In Boyden chamber assays, cells were treated with TNF- $\alpha(10 \mathrm{ng} / \mathrm{mL})$ or the control PBS and incubated for $24 \mathrm{~h}$. Cells migrating to the lower side of the filter were counted. The number of migrating cells in the TNF- $\alpha$ treated group was significantly larger than control $(P<0.05$, Student $t$-test). Experiments for each treatment were performed in triplicate. Data are reported as means $\pm \mathrm{SD}$. $B, \mathrm{MCF}-7$ cells were treated with TNF- $\alpha$ or the control. Protein was isolated 24 $\mathrm{h}$ later, and the levels of vimentin were determined by Western blot. TNF- $\alpha$ significantly increased the expression of vimentin in MCF-7 cells. Results are representative of two independent experiments.

Figure 2. Tumor necrosis factor$\alpha($ TNF- $\alpha)$ increased the expression of Snail in a nuclear factor kappa B (NFאB)-dependent way. $A$, MCF-7 cells were transfected with $300 \mathrm{ng} 3 \mathrm{X} \kappa \mathrm{B}$ luc and $50 \mathrm{ng}$ pBIND. Ten nanograms $/ \mathrm{mL}$ TNF- $\alpha, 50 \mu \mathrm{M} \mathrm{H}_{2} \mathrm{O}_{2}, 5 \mathrm{mM}$ aspirin, $5 \mathrm{mM} \mathrm{N}$-acetyl cysteine (NAC) or different combinations and the control agents were added to the indicated wells $4 \mathrm{~h}$ post-transfection, and cells were harvested $24 \mathrm{~h}$ after transfection for the luciferase activity test. Aspirin (5 mM) potently blocked the TNF- $\alpha$ - and $\mathrm{H}_{2} \mathrm{O}_{2}$-induced activation of $\mathrm{NF} \kappa \mathrm{B}(\mathrm{P}<0.05)$. NAC had no obvious effect on the activation of $N F \kappa B$ induced by TNF- $\alpha$. Experiments for each treatment were performed in triplicate. Results are reported as means $\pm \mathrm{SD}$. $B$, MCF-7 cells were pretreated with aspirin or NAC for $2 \mathrm{~h}$ or control. Cells were then incubated with TNF- $\alpha$ or control. RNA was isolated 24 $\mathrm{h}$ later, and the levels of Snail and glyceraldehyde-3-phosphate dehydrogenase (GAPDH) were determined by RT-PCR. Similar results were obtained in three independent experiments. $C$, MCF-7 cells were co-transfected with Snail promoter reporter, the internal control vector, and the IKB mutant or the control vector pcDNA3.1 and treated with TNF- $\alpha 6 \mathrm{~h}$ after transfection. Results showed that the IKB mutant nearly blocked the induction of Snail promoter induction $(P<0.05$, Student $t$-test). Experiments for each treatment were performed in triplicate. Data are reported as means \pm SD.
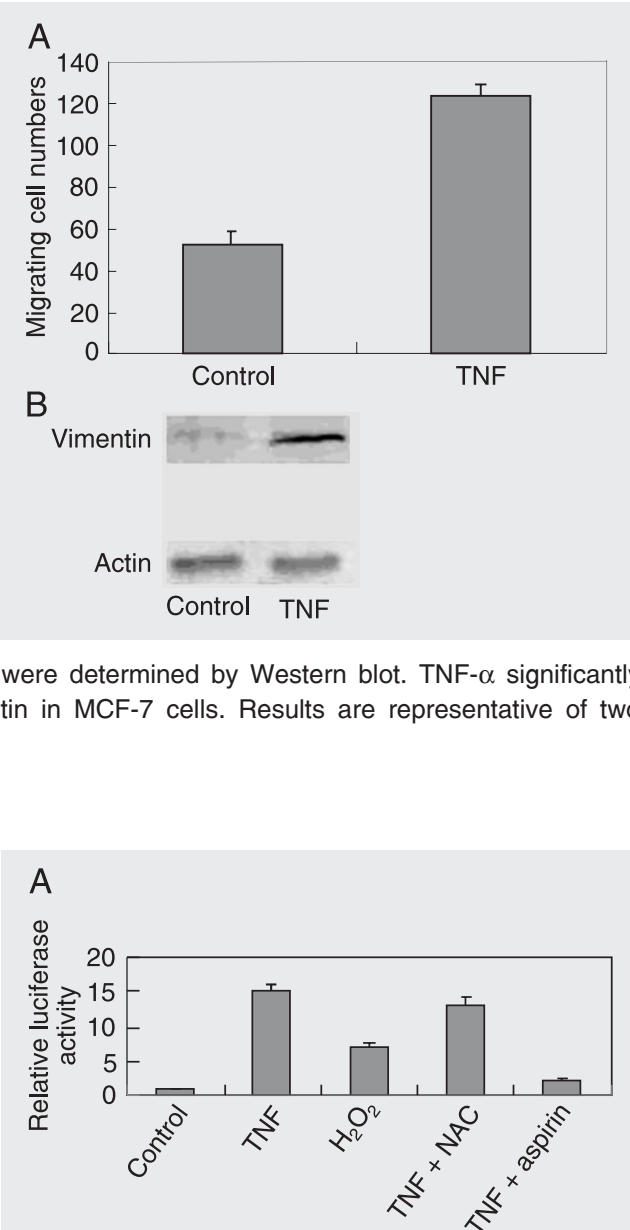

B

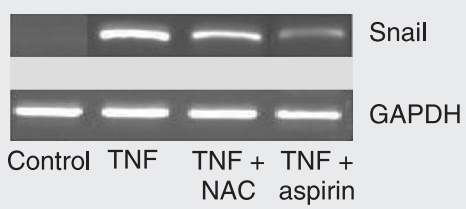

C

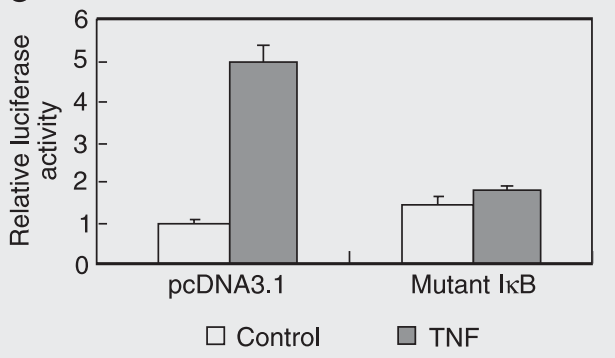

\section{TNF- $\alpha$ increased the transcription of Snail in} an NFKB-dependent way

In light of the important roles of NFKB and ROS in EMT, we raised the possibility that $\mathrm{NF} \kappa \mathrm{B}$ or ROS function importantly in the migration of MCF-7 cells induced by TNF- $\alpha$. It has also been reported that a reduced level of E-cadherin expression has a pivotal role in EMT. Thus, we proposed that NFKB or ROS may bridge TNF- $\alpha$ and EMT through E-cadherin. To examine this possibility, we tested the expression level of Snail, the transcription repressor of E-cadherin, in the different treatments. We treated cells with the combination of TNF- $\alpha$ plus NAC or aspirin, which were supposed to inhibit the production of ROS and the activation of $\mathrm{NF \kappa B}$, respectively. First, the effect of NAC and aspirin was confirmed by the $3 \mathrm{X} \kappa \mathrm{B}$ luc reporter assay. As shown in Figure 2A, NAC had little effect on NFKB activity while aspirin almost totally blocked the activation of NFאB induced by TNF- $\alpha$. The results in Figure $2 \mathrm{~B}$ reveal that the transcription of Snail was enhanced by TNF- $\alpha$ and can be inhibited by the NFKB inhibitor aspirin while it cannot be inhibited by NAC, pointing to the possibility that NFKB was the main mediator. In contrast, $\mathrm{H}_{2} \mathrm{O}_{2}$ induced up-regulation of Snail which was only mildly inhibited by aspirin (data not shown). Since aspirin has other functions in addition to being an inhibitor of activated NFKB, we also car- 
ried out the following experiment. Snail promoter was constructed and transfected into MCF-7 cells. As shown in Figure 2C, TNF$\alpha$ significantly enhanced the promoter activity of Snail which can be blocked by cotransfection with the mutant IKB expression vector. These data are consistent with previous reports showing that the Snail promoter is regulated by NFkB (15). Finally, we observed the expression of E-cadherin both at the mRNA level and at the protein level. In agreement with the fluctuating expression of Snail, a reverse trend to expression of Ecadherin was observed both at the mRNA and protein level (Figure $3 \mathrm{~A}$ and $\mathrm{B}$ ). In addition, $\mathrm{H}_{2} \mathrm{O}_{2}$ induced down-regulation of E-cadherin which was only mildly inhibited by the NFKB inhibitor. Notably, the present results do not exclude a direct effect of activated NFKB on E-cadherin itself, which may occur through loss of Snail function.

\section{Inhibition of NFKB blocked the TNF- $\alpha$ - induced migrating ability of MCF-7 cells}

On the basis of the above data, we further tested the effects of aspirin and NAC on the TNF- $\alpha$-induced EMT in MCF-7 cells and observed that it was aspirin rather than NAC that blocked the enhanced migrating ability of MCF-7 cells induced by TNF- $\alpha$. In contrast, the increased migrating ability induced by $\mathrm{H}_{2} \mathrm{O}_{2}$ was only mildly inhibited by the NFKB inhibitor aspirin (Figure 4).

\section{Discussion}

The initial process in the metastatic spread of breast carcinomas involves invasion by malignant cells through the extracellular matrix of a basement membrane, followed by their migration into lymphatic or vascular channels (16). The acquisition of migratory properties and weakening of cell-cell adhesion are imperative for tumor cell metastasis.

The continued expression and functional activity of E-cadherin are required for cells to remain tightly associated in the epithelium. In the absence of E-cadherin, many other cell adhesion and cell junction proteins expressed in epithelial cells are unable to support intercellular adhesion. The central role of E-cadherin in epithelia is demonstrated by the fact that loss of either its expression or function results in the dissolution of the epithelial architecture and the acquisition of a mesenchymal phenotype. This process, referred to as the EMT, occurs within the context of development and tu-

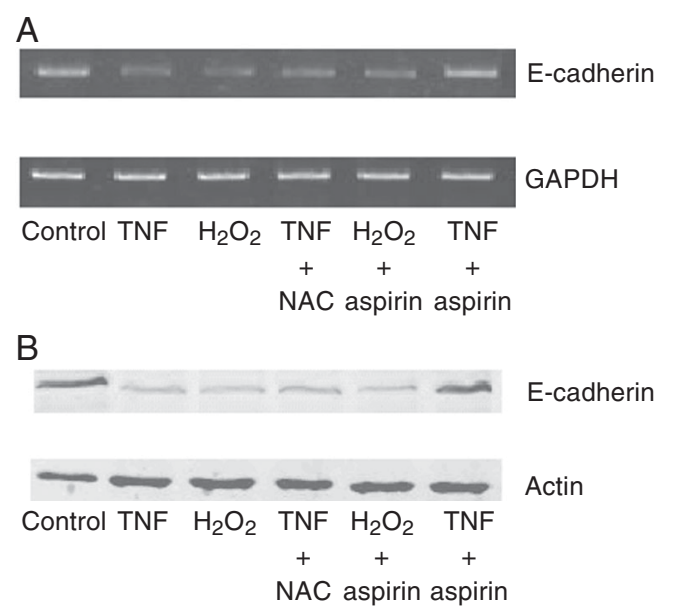

Figure 3. Tumor necrosis factor$\alpha(\mathrm{TNF}-\alpha)$ decreased the expression of $\mathrm{E}$-cadherin in a nuclear factor kappa B (NFкB)-dependent way. MCF-7 cells were pretreated with aspirin, $\mathrm{N}$-acetyl cysteine (NAC) or the control agent for $2 \mathrm{~h}$. Cells were then incubated with TNF- $\alpha, \mathrm{H}_{2} \mathrm{O}_{2}$ or control. RNA and protein were isolated $24 \mathrm{~h}$ later, and the levels of E-cadherin and glyceraldehyde-3-phosphate dehydrogenase (GAPDH) were determined by RT-PCR (A) and Western blot (B). Similar results were obtained in three independent experiments.

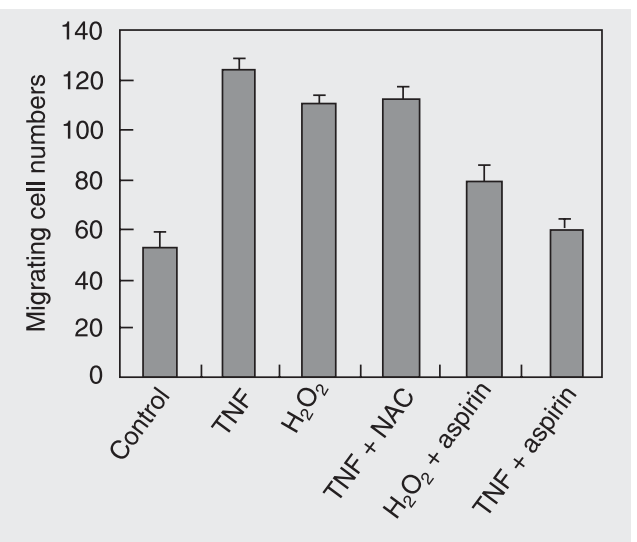

Figure 4. Inhibition of nuclear factor kappa B (NFkB) but not of reactive oxygen species prevents tumor necrosis factor- $\alpha$ (TNF- $\alpha$ )-induced migration. In Boyden chamber assays, MCF7 cells were pretreated with aspirin, $\mathrm{N}$-acetyl cysteine (NAC) or control agent for $2 \mathrm{~h}$ in the volume indicated in Material and Methods. Cells were then incubated with TNF- $\alpha, \mathrm{H}_{2} \mathrm{O}_{2}$ or control for an additional $24 \mathrm{~h}$. Cells migrating to the lower side of the filter were counted. TNF- $\alpha$ induced MCF-7 migration was inhibited by the NFKB inhibitor aspirin $(\mathrm{P}<0.05$, Student $t$-test). Inhibition of reactive oxygen species production by NAC did not alter TNF- $\alpha$-induced migration ( $\mathrm{P}>0.05$, Student $t$-test). $\mathrm{H}_{2} \mathrm{O}_{2}$-induced migration was only moderately inhibited by the inhibition of NFKB $(P<0.05$, Student $t$-test). Experiments for each treatment were performed in triplicate. Data are reported as means $\pm S D$. 
mor progression (17).

Although MCF-7 breast cancer cells are poorly invasive (18), we show that exposure of these cells to TNF- $\alpha$ leads to increased motility. NFKB plays a central role in the EMT. It has been reported that NFkB can facilitate EMT through transcriptional regulation of Snail, a transcriptional repressor of E-cadherin (12). It has also been reported that ROS could also promote EMT by activating Rac and the downstream cascade (19). ROS and NFKB can cross-talk with each other (20). Cytotoxic ROS signaling appears to be mediated in part by activation of the cJun-N-terminal kinase mitogen-activated protein kinase cascade, while in some systems ROS lead to activation of NFKB. Remarkably, new evidence has unveiled the existence of a reciprocal negative control that NFאB exerts on ROS and the c-Jun-Nterminal activities ROS and NFKB have a complicated interaction in the TNF- $\alpha$ signaling. On the one hand, NFKB can inhibit ROS production and on the other ROS can function both as an inhibitor and as an activator of NFKB depending on the context, complicating the roles of ROS and NFKB in the TNF- $\alpha$-induced EMT. In the present study, we observed that NFKB plays a vital role in this process through its transcriptional regulation of the expression of Snail and then of E-cadherin. We cannot rule out the possibility that other factors function importantly in the TNF- $\alpha$-induced EMT, such as cyclooxygenase-2 (COX-2), an inducible enzyme involved in prostaglandin (including prostaglandin E2) biosynthesis, which is overexpressed in several epithelial malignancies including breast cancer and up-regulated under the NFKB signal, while COX-2 plays an important role in metastasis (21-23). It is also possible that other proteins affected by NFKB are vital for EMT and that other factors such as protein kinase or other signal molecules function downstream from, or synergistic or parallel with, the NFKB pathway. All of these hypotheses still need to be tested. The ROS caused by TNF- $\alpha$ seemed to play a minor role in the TNF- $\alpha$ induced EMT of MCF-7 cells, though ROS can promote EMT by themselves, suggesting a complicated role of ROS in EMT. On the one hand, this can be explained by the fact that the ROS produced by TNF- $\alpha$ are different in quantity, time and compartment from those produced by treatment with exogenous $\mathrm{H}_{2} \mathrm{O}_{2}$ or under hypoxia or other conditions. On the other hand, ROS may function as activators, bystanders, or inhibitors of EMT in different situations. In some situations ROS produced by TNF- $\alpha$ act as inhibitors of NFKB by a mechanism whose details still need to be elucidated. In addition, the present study suggested that the NFKB inhibitor aspirin nearly blocked the TNF- $\alpha$-induced EMT, suggesting that NFKB may be the converging point of the complicated signal pathway induced by TNF- $\alpha$ in the process of tumor metastasis. In agreement with previous study showing that inhibition of NFKB would significantly inhibit the migrating ability of tumor cells $(24,25)$, our result may also provide valuable information for the role of aspirin in inflammation-associated tumors for the prevention of metastasis. However, it is important to note that aspirin is not a specific inhibitor of $\mathrm{NF} \kappa \mathrm{B}$, but COX-2 is also blocked by aspirin which plays an important role in tumor metastasis. Thus, we cannot rule out the possible role of COX-2 inhibition among the effects of aspirin on TNF- $\alpha$-induced EMT. Further studies are needed to detect the basal level and induced level of COX-2 in MCF-7 cells with or without aspirin treatment to answer this question. In contrast to the common inhibitory role of aspirin against $\mathrm{NF \kappa B}$, results of activation of $\mathrm{NFKB}$ signaling by aspirin have been recently reported (26). Reasons for this inconsistency might be the differences in the dose of aspirin administered and the different models used in the experiments. Further study is needed to confirm this possibility. 
The present study also pointed out that $\mathrm{H}_{2} \mathrm{O}_{2}$ alone can promote EMT in a way different from TNF- $\alpha$-induced EMT, in which $\mathrm{NF} \kappa \mathrm{B}$ only plays a minor role. Since EMT can be affected by many signal pathways and kinds of transcription factors (17), another transcription factor(s) or signal pathway(s) may be the leading factor(s) of EMT induced by ROS. A study on how ROS promote EMT is currently underway. This result also sheds light on the mechanism of tumor metastasis since hypoxia is another characteristic of tumors due to overprolifer- ation and nutrient insufficiency, which in turn produce ROS. Thus, antioxidants may also be useful for the prevention of cancer.

Our results imply a critical role for TNF$\alpha$ in stimulating EMT, in which NFkB functions essentially via the transcriptional upregulation of Snail and thus the inhibition of E-cadherin, while in ROS-induced EMT $\mathrm{NF} \kappa \mathrm{B}$ does not seem to be so essential. These data indicate the importance of determining different drug strategies for the prevention of cancer metastasis induced by different stimuli.

\section{References}

1. Bissell MJ, Radisky D. Putting tumours in context. Nat Rev Cancer 2001; 1: 46-54.

2. Leek RD, Lewis CE, Whitehouse R, Greenall M, Clarke J, Harris AL. Association of macrophage infiltration with angiogenesis and prognosis in invasive breast carcinoma. Cancer Res 1996; 56: 46254629.

3. Etoh T, Shibuta K, Barnard GF, Kitano S, Mori M. Angiogenin expression in human colorectal cancer: the role of focal macrophage infiltration. Clin Cancer Res 2000; 6: 3545-3551.

4. Moncada S, Palmer RM, Higgs EA. Nitric oxide: physiology, pathophysiology, and pharmacology. Pharmacol Rev 1991; 43: 109-142.

5. Thomsen LL, Miles DW. Role of nitric oxide in tumour progression: lessons from human tumours. Cancer Metastasis Rev 1998; 17 : 107-118.

6. Huber MA, Beug H, Wirth T. Epithelial-mesenchymal transition: NFkappaB takes center stage. Cell Cycle 2004; 3: 1477-1480.

7. Rhyu DY, Yang Y, Ha H, Lee GT, Song JS, Uh ST, et al. Role of reactive oxygen species in TGF-beta1-induced mitogen-activated protein kinase activation and epithelial-mesenchymal transition in renal tubular epithelial cells. J Am Soc Nephrol 2005; 16: 667-675.

8. Huber MA, Azoitei N, Baumann B, Grunert S, Sommer A, Pehamberger $\mathrm{H}$, et al. NF-kappaB is essential for epithelial-mesenchymal transition and metastasis in a model of breast cancer progression. $J$ Clin Invest 2004; 114: 569-581.

9. Chua HL, Bhat-Nakshatri P, Clare SE, Morimiya A, Badve S, Nakshatri H. NF-kappaB represses E-cadherin expression and enhances epithelial to mesenchymal transition of mammary epithelial cells: potential involvement of ZEB-1 and ZEB-2. Oncogene 2007; 26: 711-724.

10. Radisky DC, Levy DD, Littlepage LE, Liu H, Nelson CM, Fata JE, et al. Rac1b and reactive oxygen species mediate MMP-3-induced EMT and genomic instability. Nature 2005; 436: 123-127.

11. Bubici C, Papa S, Pham CG, Zazzeroni F, Franzoso G. NF-kappaB and JNK: an intricate affair. Cell Cycle 2004; 3: 1524-1529.

12. Bates RC, Mercurio AM. Tumor necrosis factor- $\alpha$ stimulates the epithelial-to-mesenchymal transition of human colonic organoids. Mol Biol Cell 2003; 14: 1790-1800.
13. Pennisi PA, Barr V, Nunez NP, Stannard B, Le Roith D. Reduced expression of insulin-like growth factor I receptors in MCF-7 breast cancer cells leads to a more metastatic phenotype. Cancer Res 2002; 62: 6529-6537.

14. Bachelder RE, Yoon SO, Franci C, de Herreros AG, Mercurio AM. Glycogen synthase kinase-3 is an endogenous inhibitor of Snail transcription: implications for the epithelial-mesenchymal transition. J Cell Biol 2005; 168: 29-33.

15. Barbera MJ, Puig I, Dominguez D, Julien-Grille S, Guaita-Esteruelas S, Peiro S, et al. Regulation of Snail transcription during epithelial to mesenchymal transition of tumor cells. Oncogene 2004; 23: 73457354.

16. Beavon IR. The E-cadherin-catenin complex in tumour metastasis: structure, function and regulation. Eur J Cancer 2000; 36: 16071620.

17. Thiery JP. Epithelial-mesenchymal transitions in tumour progression. Nat Rev Cancer 2002; 2: 442-454.

18. Bae SN, Arand G, Azzam H, Pavasant P, Torri J, Frandsen TL, et al. Molecular and cellular analysis of basement membrane invasion by human breast cancer cells in Matrigel-based in vitro assays. Breast Cancer Res Treat 1993; 24: 241-255.

19. Larue L, Bellacosa A. Epithelial-mesenchymal transition in development and cancer: role of phosphatidylinositol 3' kinase/AKT pathways. Oncogene 2005; 24: 7443-7454.

20. Bubici C, Papa S, Dean K, Franzoso G. Mutual cross-talk between reactive oxygen species and nuclear factor-kappa B: molecular basis and biological significance. Oncogene 2006; 25: 6731-6748.

21. Singh B, Berry JA, Shoher A, Ramakrishnan V, Lucci A. COX-2 overexpression increases motility and invasion of breast cancer cells. Int J Oncol 2005; 26: 1393-1399.

22. Ackerman WE, Zhang $\mathrm{XL}$, Rovin $\mathrm{BH}$, Kniss DA. Modulation of cytokine-induced cyclooxygenase 2 expression by PPARG ligands through NFkappaB signal disruption in human WISH and amnion cells. Biol Reprod 2005; 73: 527-535.

23. Mann JR, Backlund MG, DuBois RN. Mechanisms of disease: Inflammatory mediators and cancer prevention. Nat Clin Pract Oncol 2005; 2: 202-210. 
24. Katerinaki E, Haycock JW, Lalla R, Carlson KE, Yang Y, Hill RP, et al. Sodium salicylate inhibits TNF- $\alpha$-induced NF-kappaB activation, cell migration, invasion and ICAM-1 expression in human melanoma cells. Melanoma Res 2006; 16: 11-22.

25. Ichikawa $\mathrm{H}$, Takada $\mathrm{Y}$, Murakami A, Aggarwal BB. Identification of a novel blocker of I kappa B $\alpha$ kinase that enhances cellular apoptosis and inhibits cellular invasion through suppression of NF-kappa Bregulated gene products. J Immunol 2005; 174: 7383-7392.

26. Stark LA, Reid K, Sansom OJ, Din FV, Guichard S, Mayer I, et al. Aspirin activates the NF-\{kappa\}B signalling pathway and induces apoptosis in intestinal neoplasia in two in vivo models of human colorectal cancer. Carcinogenesis 2006. 